\title{
Trapianto di rene in pazienti con patologia policistica: valutazione pre-trapianto e risultati. Esperienza italiana
}

\author{
G. Mosconi, E. Persici, A. Ricci, C. Kwin Kwedi, A. Nanni Costa, S. Stefoni \\ Unità Operativa di Nefrologia Dialisi Trapianto Policlinico S. Orsola di Bologna, Bologna \\ Centro Nazionale Trapianti, Roma
}

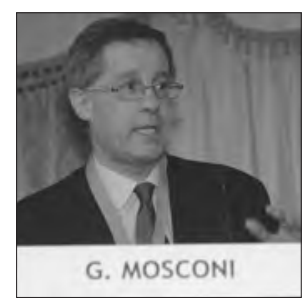

\section{Introduzione}

La malattia policistica renale autosomica dominante (ADPKD) rappresenta la causa più frequente di insufficienza renale cronica su base genetica con una incidenza compresa tra $1 / 500$ e $1 / 1000$ nati vivi. La $\mathrm{ADPKD}$ è una malattia ereditaria monogenica caratterizzata da mutazioni genetiche che possono interessare due diversi geni: PKD1 (ADPKD tipo 1, 8590\% dei casi), mappato sul cromosoma 16p13,3 e PKD2 (ADPKD tipo 2, 10-15\% dei casi) mappato sul cromosoma 4q21 (1). Questi due geni codificano per due proteine di membrana (policistina-1 e policistina-2) localizzate sulla membrana e sulle cilia del tubulo renale e dell'epitelio biliare $(2,3)$. L'ADPKD può essere inquadrata nell'ambito delle ciliopatie (4). La ADPKD tipo 1 si manifesta clinicamente nell'età giovane-adulta (tra i 30 e 40 anni), mentre il tipo 2 ha un esordio più tardivo e una più lenta progressione verso l'insufficienza renale (5). È riportata un'incidenza annuale d'insufficienza renale terminale (IRC) da malattia policistica variabile tra il 4 e il 9\% (USA 8,7\% negli uomini e 6,9\% nelle donne, Europa 7,8\% negli uomini e $6 \%$ nelle donne, Giappone 5,6\% negli uomini e del $4 \%$ nelle donne) (1). I dati del Registro Italiano Dialisi e Trapianto relativi al 2008 mostrano che 1'8,2\% dei pazienti con indicazione alla dialisi o al trapianto renale è affetto da malattia policistica autosomica dominante. L'ADPKD è una malattia sistemica con possibili manifestazioni extrarenali; lo sviluppo di cisti renali frequentemente si associa a formazioni cistiche in altri organi (fegato, pancreas, milza), a ipertensione arteriosa, a dilatazioni aneurismatiche (intracraniche, aortiche, polmonari, spleniche, pancreatiche), a difetti valvolari cardiaci, a diverticolosi del colon, a ernie addominali o inguinali, ad ectasia biliare. I pazienti con rene policistico, nonostante le manifestazioni extrarenali correlate, hanno una sopravvivenza in dialisi maggiore rispetto ai pazienti affetti da insufficienza renale terminale da altre cause $(6,7)$. Anche relativamente a programmi di trapianto i risultati riportati in letteratura risultano mediamente superiori a quelli che si riscontrano in altre patologie renali (nefropatie glomerulari o vascolari). Il potenziale coinvolgimento di altri organi richiede una particolare attenzione nella fase di studio per programmi di trapianto. Spesso si registrano atteggiamenti diversi tra i vari Centri Trapianto per quanto riguarda gli aspetti chirurgici (indicazione alla nefrectomia) e la tipologia di indagini strumentali aggiuntive (per esempio, studio vascolare cerebrale); la proposta di percorsi differenziati per l'inserimento operativo in lista può ingenerare difficoltà di gestione da parte dei Centri Dialisi che hanno in carico i pazienti. Anche in letteratura, peraltro, si evince un'ampia eterogeneità di comportamenti.

$\mathrm{Al}$ fine di individuare alcuni punti di riferimento lo studio si è proposto di verificare l'atteggiamento dei diversi Centri Trapianto Italiani relativamente alla gestione dei pazienti policistici candidati al trapianto sia dal punto di vista chirurgico (nefrectomia) che diagnostico/ strumentale (indagini al momento dell'inserimento in lista e nel successivo follow-up). È stato inoltre verificato, in collaborazione con il Centro Nazionale Trapianti, l'impatto della patologia policistica sui programmi di trapianto e i relativi risultati a breve, medio e lungo termine. 


\section{Pazienti e Metodi}

Al fine di valutare le strategie dei Centri Italiani Trapianto è stato elaborato un questionario costituito da 8 domande relative ai criteri per l'inserimento e per il monitoraggio dei pazienti con patologia policistica nelle liste di attesa di trapianto da donatore cadavere. I quesiti si sono focalizzati su aspetti chirurgici quali l'eventuale nefrectomia preventiva indipendentemente da problematiche cliniche, sulle principali indicazioni alla nefrectomia in caso di atteggiamento conservativo (ingombro addominale, infezioni, coliche/ematuria) e sui relativi tempi di intervento (prima dell'inserimento in lista attiva, al momento o dopo il trapianto). Sono state inoltre verificate le indagini strumentali richieste per l'inserimento in lista e per il monitoraggio durante il periodo di attesa (indagini radiologiche per studio dimensioni renali, ricerca di aneurismi cerebrali). Nel questionario è stato inoltre richiesto se la patologia policistica costituisse una controindicazione a programmi di doppio trapianto renale; è stata infine valutata l'esperienza dei Centri italiani in merito ai programmi di trapianto combinato fegato-rene.

Per un'analisi dell'impatto della patologia policistica sui programmi di trapianto sono stati analizzati, in collaborazione con il Centro Nazionale Trapianti e utilizzando il database del sistema informativo (SIT), le caratteristiche e i risultati dei trapianti effettuati in Italia nel periodo gennaio 2002-dicembre 2008. L'elaborazione è stata fatta sui dati disponibili al 30 aprile 2011; il periodo di osservazione variava da 16 a 112 mesi con un aggiornamento del follow-up a 12 mesi superiore al $97 \%$. Complessivamente sono stati analizzati i dati di 11.412 trapiantati (9.995 pazienti sottoposti a primo trapianto renale singolo o doppio trapianto, 114 sottoposti a trapianto combinato fegato/rene, 1303 sottoposti a trapianto da donatore vivente) come riportato in Tabella I; sono stati esclusi i pazienti con secondo trapianto. È stato valutato l'impatto numerico della patologia policistica nell'ambito dei diversi programmi di trapianto. L'andamento del trapianto singolo o doppio

TABELLA I - CASISTICA COMPLESSIVA ANALIZZATA

\begin{tabular}{lccc}
\hline & $\begin{array}{c}\text { Primo Tx renale } \\
\text { (singolo o doppio) }\end{array}$ & $\begin{array}{c}\text { Tx combinato } \\
\text { fegato-rene }\end{array}$ & $\begin{array}{c}\text { Tx } \\
\text { da vivente }\end{array}$ \\
\hline Totale pz trapiantati & 9995 & 114 & 1303 \\
Policistici & $1182(12 \%)$ & $28(25 \%)$ & $92(7 \%)$ \\
Altre nefropatie & $8813(88 \%)$ & $86(75 \%)$ & $1211(93 \%)$ \\
\hline
\end{tabular}

nei pazienti policistici è stato confrontato con i dati dei pazienti affetti da altre nefropatie (sopravvivenza organo, sopravvivenza paziente); l'analisi è stata condotta valutando le caratteristiche anagrafiche al momento del trapianto, l'età dei donatori, i tempi di ischemia fredda, il grado di immunizzazione pre-trapianto, i tempi di attesa in lista. Per lo stesso periodo di osservazione è stato valutato l'outcome dei 114 pazienti sottoposti a trapianto combinato fegato-rene. Relativamente al trapianto da donatore vivente (1303 trapianti eseguiti in Italia nel periodo 2001-2010) l'attenzione è stata focalizzata sulle caratteristiche dei donatori (familiarità, utilizzo di donatori potenzialmente a rischio di patologia policistica).

\section{Analisi statistica}

I dati analizzati sono stati estratti dal SIT in data 30 aprile 2011 tramite l'ausilio del software Busness Objects ver 5.1.2. Tutte le analisi sono state eseguite tramite l'utilizzo di STATA ver 10.0. Le variabili qualitative sono state espresse come percentuali e le variabili continue come media e deviazione standard. Il confronto tra le variabili qualitative è stato fatto utilizzando il test chi-quadro, mentre per le variabili continue è stato utilizzato il t-test di Student. L'analisi della sopravvivenza dei pazienti è stata valutata mediante il metodo di Kaplan-Meier e confrontato con il Log-Rank test. I valori di $\mathrm{p}<0,05$ sono stati considerati statisticamente significativi.

\section{Risultati}

Complessivamente si è registrata un'ottima collaborazione dei Centri Trapianto; 35 dei 38 Centri (Fig. 1) hanno risposto a tutte le domande del questionario (adesione del 92\%).

Per quanto riguarda l'indicazione alla nefrectomia è emerso un atteggiamento prevalentemente conservativo (nefrectomia solo in presenza di problemi clinici) nel $91 \%$ dei Centri (32/35). Nei 3 Centri nei quali viene eseguita di prassi la nefrectomia prima dell'inserimento in lista (indipendentemente dalla presenza di problematiche cliniche) in 2 viene proposta la mononefrectomia, in 1 la binefrectomia. Nei 32 Centri con atteggiamento conservativo la principale indicazione alla nefrectomia è costituita dall'ingombro addominale (58\% dei Centri), seguita dalla comparsa di coliche e/o di ematuria (25\% dei Centri) e infine dalla ricorrenza di infezioni urinarie (3\% dei Centri). Relativamente ai tempi chirurgici, sem- 


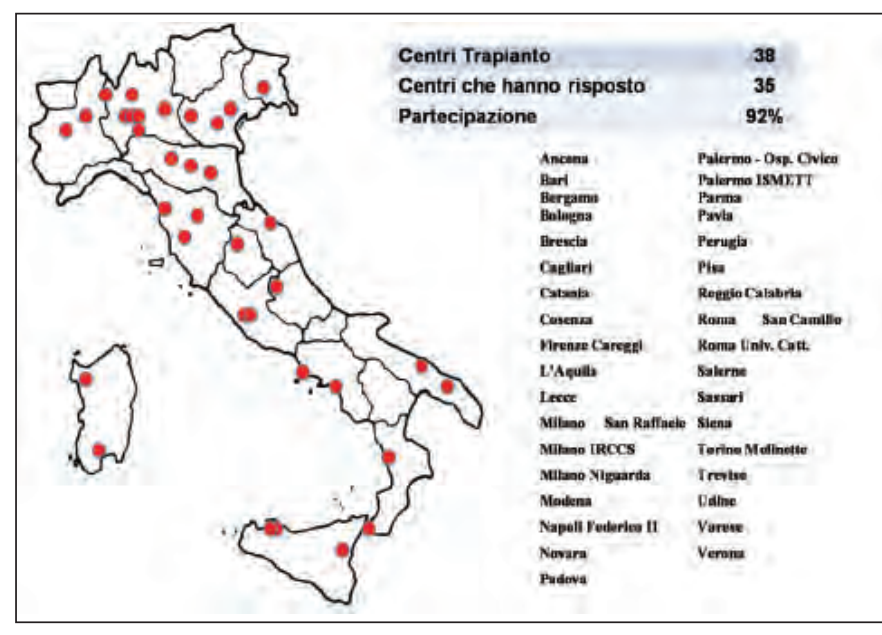

Figura 1 - Centri Trapianto Italiani che hanno risposto al questionario.

pre nei Centri con approccio conservativo, la nefrectomia viene effettuata nel $83 \%$ dei casi durante il periodo di permanenza in lista; nel restante $17 \%$, qualora i problemi siano tecnicamente procrastinabili, viene praticata contemporaneamente al trapianto. L'atteggiamento riportato si riferisce a programmi di trapianto da donatore cadavere; nel trapianto da vivente viene segnalata una maggior tendenza alla nefrectomia contemporanea al trapianto, soprattutto in presenza di una diuresi conservata. Relativamente alle indagini strumentali mirate alla definizione dell'entità dell'ingombro addominale, l's6\% dei Centri associa l'ecografia associata alla tomografia computerizzata ( $46 \%$ senza mezzo di contrasto, $40 \%$ con mezzo di contrasto); per il restante $14 \%$ dei Centri è sufficiente, in assenza di altre problematiche, l'indagine ecografica. Il monitoraggio durante la permanenza in lista d'attesa è effettuato di routine tramite esame ecografico dell'addome ogni 1-2 anni nel 72\% dei Centri e nel restante $28 \%$ associando all'ecografia un'indagine TC. Anche in questo caso la presenza di diuresi residua condiziona le relative procedure (TC con o senza mezzo di contrasto). Dal questionario emerge che indagini radiologiche (RM o TC) per la ricerca di aneurismi o malformazioni vascolari cerebrali sono richieste sempre nel $74 \%$ dei Centri, mentre nel 23\% solo in presenza di anamnesi familiare per emorragia cerebrale; il 3\% dei Centri non ritiene indicato procedere con indagini supplementari. La maggior parte dei Centri propone inoltre l'esecuzione di uno studio del grosso intestino (colonscopia o Rx clisma opaco) indipendentemente dall'età o dalla presenza di sangue occulto. La presenza di patologia policistica non costituisce una controindicazione assoluta per programmi di doppio trapianto renale per nessuno dei 28

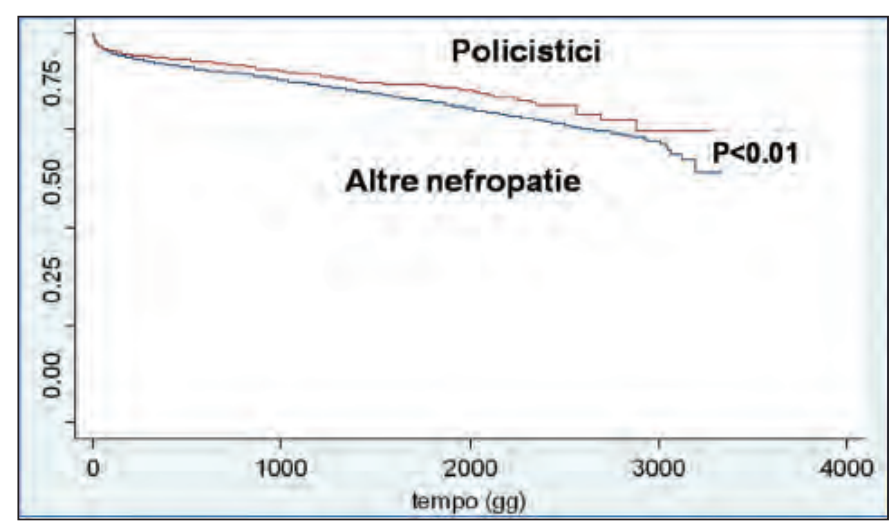

Figura 2 - Primo trapianto di rene (singolo o doppio). Sopravvivenza del graft nei pazienti affetti da ADPKD rispetto ai pazienti con altre nefropatie.

Centri che svolgono tale attività (in particolare nessuna controindicazione per 11 , controindicazione relativa per 17 Centri); 7 Centri dichiarano di non possedere alcuna esperienza nel programma di doppio trapianto renale. Infine per quanto riguarda il trapianto combinato fegatorene 7 Centri propongono regolarmente tale programma; i restanti Centri hanno riportato esperienze sporadiche o nessuna esperienza. Le risposte ai diversi quesiti sono riportate in maniera riassuntiva nella Tabella II. Nell'ambito dei 9.995 pazienti sottoposti a primo trapianto di rene (rene singolo o doppio trapianto) i pazienti affetti da patologia policistica costituiscono il $12 \%$ della casistica (n. 1182). Nel gruppo di pazienti policistici la percentuale di pazienti di sesso femminile è del $42 \%$, nel gruppo con altre nefropatie del $35 \%$. La percentuale di doppi trapianti risulta del tutto sovrapponibile $(6,2 \%$ policistici, $6,1 \%$ altre nefropatie).

A un follow-up compreso fra 16 e 112 mesi i pazienti con patologia policistica presentano una miglior sopravvivenza del graft rispetto ai pazienti con altre nefropatie (a 1, 3, 5 e 8 anni rispettivamente del $93,89,86,75 \%$ rispetto a $92,87,82,73 \%$ ) come riportato in Figura 2; la differenza risulta statisticamente significativa $(\mathrm{p}<0,01)$. Il dato assume maggior rilevanza se valutato in rapporto all'età che risulta maggiore nei pazienti policistici $(52 \pm 9$ anni rispetto a $47 \pm 14$ anni) e nei relativi donatori (51 \pm 15 anni rispetto $46 \pm 18$ anni). Nessuna differenza è emersa in termini di ischemia fredda $(14,8 \pm 6,7$ ore policistici, $14,7 \pm 5,9$ altre nefropatie). Non sono state riscontrate differenze circa il grado di immunizzazione pre-trapianto con un PRA di $1 \pm 8$ nei policistici, di $2 \pm 10$ nelle restanti nefropatie; la percentuale di pazienti trapiantati con PRA $>30$ è risultata rispettivamente dell'1,5\% e del 2,5\%. Il 


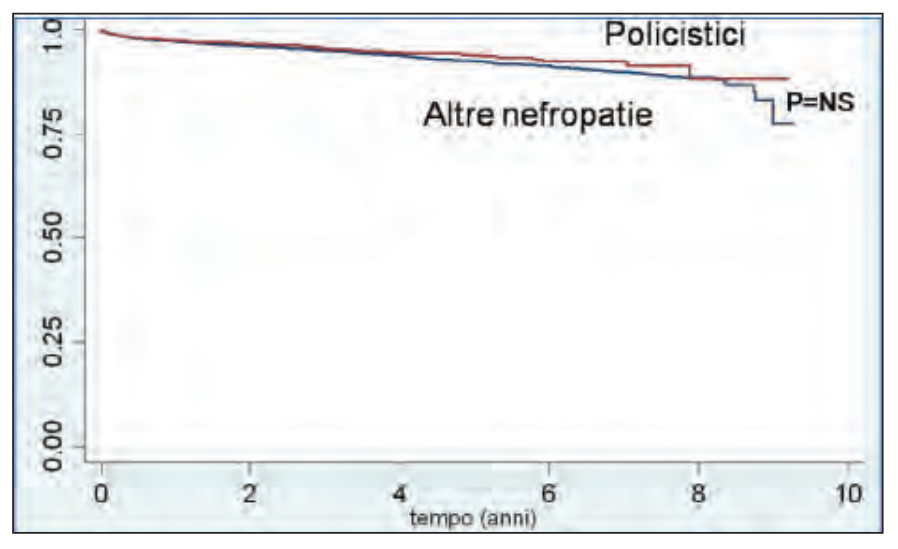

Figura 3 - Primo trapianto di rene (singolo o doppio). Sopravvivenza dei pazienti affetti da ADPKD rispetto ai pazienti con altre nefropatie.

\section{TABELLA II - RISPOSTE DEI CENTRI TRAPIANTO AI QUE- SITI DEL QUESTIONARIO}

\begin{tabular}{|c|c|}
\hline $\begin{array}{l}\text { NEFRECTOMIA PRE-TX (Atteggiamento del } \\
\text { Centro) }\end{array}$ & Numero \\
\hline Atteggiamento conservativo (nefrectomia al bisogno) & $32(91 \%)$ \\
\hline Mononefrectomia sempre & $2(6 \%)$ \\
\hline Binefrectomia sempre & $1(3 \%)$ \\
\hline Embolizzazione & 0 \\
\hline \multicolumn{2}{|l|}{ NEFRECTOMIA PRE-TX TIMING CHIRURGICI } \\
\hline Prima dell'inserimento in lista & $29(83 \%)$ \\
\hline Contemporanea al trapianto & $6(17 \%)$ \\
\hline \multicolumn{2}{|c|}{$\begin{array}{l}\text { PRINCIPALE INDICAZIONE A NEFRECTOMIA } \\
\text { (Centri Trapianto con atteggiamento conservativo) }\end{array}$} \\
\hline Ingombro addominale & $58 \%$ dei Centri \\
\hline Colica/rottura cisti (>1 episodio/anno) & $25 \%$ dei Centri \\
\hline Infez. urinarie frequenti (> 1/anno) & $3 \%$ dei Centri \\
\hline \multicolumn{2}{|l|}{ INDAGINI STRUMENTALI PRE TX } \\
\hline Eco-addome & $5(14 \%)$ \\
\hline Eco-addome + TC senza contrasto & $16(46 \%)$ \\
\hline Eco-addome + TC con contrasto & $14(40 \%)$ \\
\hline \multicolumn{2}{|c|}{ MONITORAGGIO DURANTE PERMANENZA IN LISTA } \\
\hline Eco-addome (ogni 1-2 anni) & $25(72 \%)$ \\
\hline Eco-addome + TC senza contrasto (ogni 1-2 anni) & $5(14 \%)$ \\
\hline Eco-addome + TC con contrasto (ogni 1-2 anni) & $5(14 \%)$ \\
\hline \multicolumn{2}{|c|}{$\begin{array}{l}\text { INDAGINI AGGIUNTIVE (TC O RMN X ANEURISMI } \\
\text { CEREBRALI) }\end{array}$} \\
\hline Sempre & $26(74 \%)$ \\
\hline Solo se anamnesi famigliare positiva & $8(23 \%)$ \\
\hline Mai & $1(3 \%)$ \\
\hline \multicolumn{2}{|c|}{ PATOLOGIA POLICISTICA E PROGRAMMA DI DOPPIO TX } \\
\hline Controindicazione relativa & $17(49 \%)$ \\
\hline Nessuna controindicazione & $11(31 \%)$ \\
\hline Controindicazione assoluta & 0 \\
\hline Nessuna esperienza doppio tx & $7(20 \%)$ \\
\hline \multicolumn{2}{|c|}{ ESPERIENZA IN PROGRAMMI DI TX COMBINATO } \\
\hline Nessuna esperienza & $17(49 \%)$ \\
\hline Sporadica esperienza & $11(31 \%)$ \\
\hline Regolare programma & $7(20 \%)$ \\
\hline
\end{tabular}

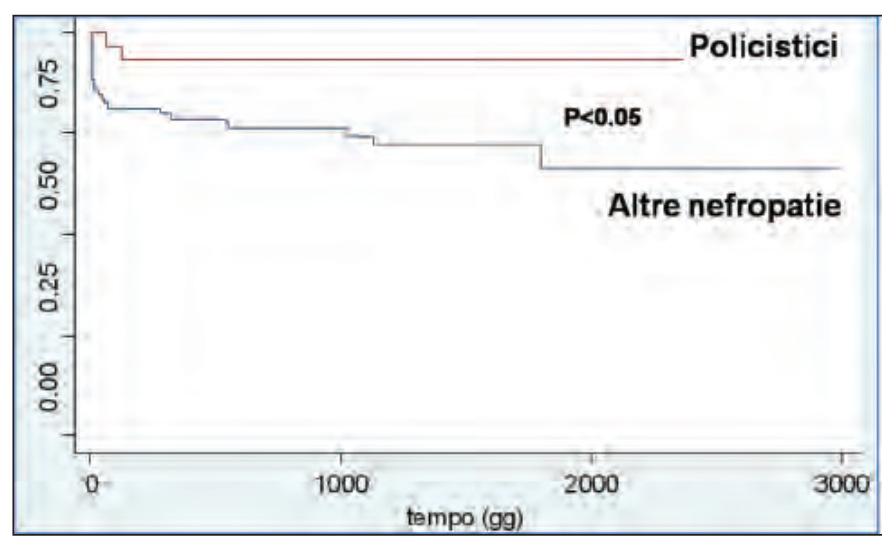

Figura 4 - Trapianto combinato fegato-rene. Sopravvivenza del rene trapiantato nei pazienti affetti da ADPKD rispetto ai pazienti con altre nefropatie.

tempo medio di attesa in lista dei pazienti con $\mathrm{ADPKD}$ è risultato lievemente inferiore a quello dei pazienti con altre nefropatie $(27 \pm 28$ mesi rispetto a $30 \pm 32$ mesi). Le caratteristiche dei due gruppi di pazienti sono riportate nella Tabella III. Relativamente alla mortalità dei pazienti i dati risultano sostanzialmente sovrapponibili nei due gruppi; a 5 anni la sopravvivenza risulta del $94 \%$ nei pazienti policistici, del $92,5 \%$ nei pazienti con altre nefropatie (Fig. 3). Fra le cause di decesso gli eventi cardiovascolari (infarto, complicanze vascolari, emorragia cerebrale, stroke) hanno un'incidenza del tutto sovrapponibile nei due gruppi (24 su 1182 nei policistici, 241 su 8813 nei pazienti con altre nefropatie) così come le infezioni batteriche (12 su 1182 rispetto a 117 su 8813). Nell'ambito dei programmi di trapianto combinato fegato-rene i pazienti con patologia policistica costituiscono il $25 \%$ della casistica $(28 / 114)$. Anche i risultati relativi a tale programma confermano un miglior andamento del rene trapiantato nei pazienti con $\mathrm{ADPKD}$ rispetto al gruppo di pazienti con altre nefropatie (a 1 anno rispettivamente $92 \%$ rispetto a $79 \%$ ); l'andamento presenta una differenza statisticamente significativa $(p<0,05)$ come riportato in Figura 4, a fronte di un'età maggiore sia dei pazienti con ADPKD sottoposti a trapianto (47 anni rispetto a 41 anni nei pazienti con altre nefropatie) che dei relativi donatori $(39 \pm 14$ anni rispetto $30 \pm 12$ anni). Il dato a lungo termine presenta il limite di un modesto numero di pazienti policistici che hanno raggiunto un follow-up pari o superiore a 5 anni ( $5 / 28$ pazienti).

Nell'ambito dei programmi di trapianto da donatore vivente effettuati nel periodo 2001-2010 la prevalenza dei pazienti policistici risulta del 7\% (92/1303); relativamente ai donatori 64 erano non consanguinei, 16 genitori e 12 fratelli o sorelle (gruppo a rischio di patologia policistica). 
TABELLA III - PRINCIPALI CARATTERISTICHE DEI PAZIENTI SOTTOPOSTI A PRIMO TRAPIANTO RENALE

\begin{tabular}{lcc}
\hline & Policistici & Altre Nefropatie \\
\hline Singolo tx renale & $1108(93,8 \%)$ & $8277(93,9 \%)$ \\
Doppio tx renale & $74(6,2 \%)$ & $536(6,1 \%)$ \\
Maschi & $685(58 \%)$ & $5728(65 \%)$ \\
Femmine & $497(42 \%)$ & $3085(35 \%)$ \\
Età ricevente & $52 \pm 9$ & $47 \pm 14$ \\
Età donatore & $51 \pm 15$ & $46 \pm 18$ \\
Tempo medio di attesa (mesi) & $27 \pm 28$ & $30 \pm 32$ \\
Tempo ischemia fredda (ore) & $14,8 \pm 6,7$ & $14,7 \pm 5,9$ \\
Ultimo PRA (\%) & $1 \pm 8$ & $2 \pm 10$ \\
\hline
\end{tabular}

\section{Discussione}

Il nostro studio conferma il significativo impatto della patologia policistica nei programmi di trapianto di rene $(12 \%$ dei pazienti sottoposti a primo trapianto di rene da donatore cadavere, $25 \%$ della casistica di trapianto combinato fegato/rene). Tale prevalenza, sostanzialmente sovrapponibile a quella riscontrata nelle liste di attesa in Emilia-Romagna, è significativamente superiore alla incidenza dei pazienti policistici che giungono annualmente alle fasi terminali della insufficienza renale; il dato può essere interpretato alla luce dell'età media di inizio dialisi e della buona risposta al trattamento dialitico, in termini di morbidità e mortalità, di questa popolazione di pazienti che favorisce, nella maggior parte dei casi, l'inserimento operativo in programmi di trapianto $(6,7)$. Dal lavoro emerge che la patologia policistica, nonostante i possibili problemi di spazio addominale, non condiziona l'iscrizione a programmi di doppio trapianto di rene. Il dato, che emerge dalle risposte al questionario (nessun Centro italiano considera la patologia policistica una controindicazione assoluta a tale programma), viene confermato dalla attività rilevata a livello nazionale che mostra una percentuale di doppi trapianti sul totale della casistica del 6,2\% nei pazienti policistici e del 6,1\% nel gruppo con altre nefropatie.

Lo studio conferma nei pazienti con patologia policistica, in analogia con altre malattie genetiche, la praticabilità di programmi di trapianto da donatore vivente (8); tali programmi dal punto di vista numerico risentono della più limitata disponibilità di consanguinei potenzialmente idonei. L'esperienza italiana dal 2001 al 2010 mostra una prevalenza di pazienti policistici trapiantati da vivente del $7 \%$ (92/1303 trapianti) rispetto al $12 \%$ riscontrato nei programmi da donatore cadavere. La maggior parte dei donatori è costituita da non consanguinei (70\%) o da genitori del ramo non affetto da patologia (17\%); solo 12 donatori (13\%) fanno parte della categoria di persone a rischio di patologia policistica (fratello o sorella). L'accuratezza diagnostica per definire la presenza di patologia policistica si avvale oggi di criteri diagnostici ben definiti e stratificati per età (9), di un continuo sviluppo delle indagini di imaging che possono ben integrarsi con analisi genetiche di linkage e/o di sequenziamento del DNA (tanto più efficaci quanto maggiore il numero di familiari affetti da patologia) in soggetti con un'età nella quale le indagini radiologiche possono non essere diagnostiche (10). E auspicabile che il progressivo affinarsi delle indagini possa facilitare programmi di donazione, oltre che da fratelli/sorelle, anche tra moglie/marito una volta accertata con accettabile attendibilità l'assenza di malattia nella prole (aspetto psicologico non secondario).

Per quanto riguarda i risultati del trapianto a mediolungo termine, come già riportato in letteratura, lo studio registra un miglior andamento funzionale del trapianto renale rispetto ai pazienti affetti da altre nefropatie, nonostante la natura sistemica della patologia policistica (11-15). Il dato assume ancor più valore se si considerano le età medie dei riceventi e dei relativi donatori, mediamente superiori nel gruppo dei pazienti policistici. I tempi di ischemia fredda ed il grado di immunizzazione sono del tutto sovrapponibili nei due gruppi; il tempo di attesa, solo lievemente inferiore nel gruppo dei pazienti policistici $(27 \pm 28$ vs $30 \pm 32$ mesi), non risulta in grado di giustificare il diverso andamento funzionale. E ipotizzabile che un ruolo di primo piano possa essere giocato dalla recidiva della nefropatia di base, ovviamente assente nei policistici, che può significativamente condizionare l'andamento funzionale dell'organo trapiantato a lungo termine. Lo studio, incentrato sulla verifica dei risultati del trapianto nei pazienti con patologia policistica, mette a confronto genericamente $\mathrm{i}$ risultati di questa popolazione con le altre nefropatie considerate globalmente; il dato potrà meglio essere analizzato dopo una ulteriore stratificazione delle diverse nefropatie.

Le curve di sopravvivenza dei pazienti non presentano differenze significative tra pazienti con APKD e pazienti con altre nefropatie; è interessante notare come l'incidenza di patologia cardiovascolare, in particolare emorragia cerebrale, e di infezioni batteriche/sepsi sia sostanzialmente sovrapponibile nei due gruppi, nonostante gli specifici fattori di rischio legati alla patologia policistica (aneurismi cerebrali, infezioni a carico dei reni nativi, possibile maggior incidenza di 
diabete mellito post-trapianto).

I dati del lavoro confermano che il trapianto combinato fegato/rene nei pazienti policistici (indicazione costituita da insufficienza renale cronica associata a ingombro addominale in gran parte legato alle dimensioni del fegato e problemi di malassorbimento) risulta oggi una consolidata e valida opzione terapeutica ( $25 \%$ di pazienti policistici sull'intera casistica nazionale). Si evince un buon andamento funzionale del rene trapiantato con una sopravvivenza superiore rispetto ai pazienti con altre nefropatie/epatopatie; il dato, sovrapponibile a quello dei programmi di trapianto renale isolato, resta da verificare su una più ampia casistica e per un più prolungato followup. Va in ogni caso considerato che, nell'ambito dei programmi di trapianto combinato, è presente un'ampia eterogeneità delle caratteristiche dei candidati; i pazienti policistici presentano al trapianto mediamente condizioni cliniche generali meno compromesse rispetto ai pazienti con altre nefropatie/epatopatie (MELD >30 nella maggioranza dei casi). Emerge peraltro la necessità di un'accurata valutazione nefrologica, epatologica e chirurgica per evitare un'indiscriminata espansione del programma e mantenere un'ottimale gestione delle risorse di organi. La nostra esperienza (16 policistici su 43 trapianti combinati fegato/rene) conferma i buoni risultati del programma; riteniamo che dal punto di vista nefrologico l'indicazione a trapianto combinato nei pazienti policistici debba essere posta in caso di regolare trattamento sostitutivo artificiale $o$, nei pazienti preemptive, in presenza di una clearance della creatinina $<35 \mathrm{ml} / \mathrm{min}$. Riteniamo inoltre opportuno evitare quadri di malnutrizione severa; lo stato nutrizionale al momento del trapianto costituisce un indice prognostico di mortalità e morbilità perioperatoria (16).

Linsieme dei risultati dei diversi programmi di trapianto (singolo rene, doppio rene, combinato fegato/rene) indirettamente evidenzia un'adeguata gestione dei pazienti policistici sia nella fase d'inserimento che di mantenimento in lista di attesa, premessa indispensabile per una riduzione delle potenziali complicanze extrarenali post-trapianto. Dalle risposte al nostro questionario si riscontra, pur in assenza di specifiche linee guida, una sostanziale uniformità di vedute circa l'opportunità di un completamento di indagini strumentali. Emerge un consenso circa l'indicazione all'esecuzione della colonscopia anche in pazienti con età inferiore a 50 anni. Complessivamente il 97\% dei Centri propone uno studio vascolare cerebrale (RM o TC); nello specifico il $64 \%$ dei Centri lo propone in tutti i pazienti policistici mentre il $23 \%$ solo in presenza di dati anamnestici di familiarità per emorragia sub-aracnoidea (ESA). Tali strategie sono in linea con i dati epidemiologici e con la più recente letteratura internazionale (17); l'emorragia da rottura di aneurismi cerebrali costituisce il 4-7\% delle cause di morte nei pazienti con ADPKD e compare solitamente in età più giovane rispetto alla popolazione generale (39 anni contro 51 anni), più frequentemente nel sesso maschile; l'incidenza di aneurismi è 4-6 volte superiore a quella della popolazione generale, in particolare nei pazienti con familiarità per emorragia sub-aracnoidea $(18,19)$. Nella procedura del nostro Centro proponiamo una indagine vascolare cerebrale (preferibilmente Angio RM) in tutti pazienti al momento dell'inserimento in lista; nei pazienti con familiarità negativa lo studio vascolare cerebrale non costituisce tuttavia un'indagine vincolante qualora il paziente, adeguatamente informato circa i vantaggi e rischi, preferisca non sottoporsi a tale accertamento. In presenza di aneurismi la decisione relativa al tipo di approccio (conservativo o interventistico, modalità di approccio) è multidisciplinare e coinvolge il Neuroradiologo ed il Neurochirurgo; nella valutazione complessiva vengono presi in considerazione sede, diametro, morfologia dell'aneurisma, possibili complicanze legate alla procedura (morbilità e mortalità), familiarità per ESA, condizioni cliniche generali e aspettativa di vita del paziente. Ovviamente ogni scelta strategica viene condivisa con il paziente e la famiglia dopo accurata informazione. Solitamente in presenza di piccoli aneurismi $(<5-7 \mathrm{~mm})$ con basso rischio di rottura l'approccio è di tipo conservativo, con follow-up radiologico (6-12 mesi). In questi pazienti si rende necessaria un'ottimale gestione dei valori pressori e, in caso di trapianto, particolare attenzione viene posta nelle fasi di induzione o di risveglio post-intervento (fasi di particolare rischio ipertensivo). L'approccio interventistico è solitamente riservato a pazienti con aneurismi di dimensioni maggiori a $10 \mathrm{~mm}$. In caso di negatività dell'indagine di screening proponiamo un controllo radiologico ogni 2-3 anni nei pazienti con familiarità positiva per ESA e ogni 5 anni negli altri pazienti.

Relativamente allo studio e al monitoraggio delle dimensioni renali 1'86\% dei Centri propone indagini di secondo livello (TC addome con o senza mezzo di contrasto), mentre solo il $14 \%$ ritiene sufficiente uno studio ecografico; l'impiego di mezzo di contrasto nell'esecuzione della TC risulta condizionato dalla persistenza o meno di una diuresi residua. La nostra procedura associa di routine un'ecografia a TC senza mezzo di contrasto; qualora resti da definire la natura di cisti renali complesse l'indagine radiologica viene completata con utilizzo di mezzo di contrasto.

Come già riportato uno degli argomenti più controversi è costituito dalle indicazioni alla nefrectomia e dai 
relativi tempi chirurgici; in questo contesto è possibile un certo margine di miglioramento. La letteratura internazionale non aiuta peraltro ad individuare un percorso ottimale; spesso i dati riportati si basano su casistiche limitate e su esperienze chirurgiche personali. Alcune scuole propongono un approccio conservativo tranne che in presenza di problemi clinici (ingombro addominale, infezioni o episodi emorragici ricorrenti) senza specificare entità dell'ingombro o numero delle complicanze che rendono opportuno procedere alla nefrectomia (20); altri gruppi riportano una miglior sopravvivenza del graft nei pazienti sottoposti a nefrectomia preventiva mono o bilaterale (21, 22), altri ancora considerano la nefrectomia posttrapianto l'intervento di scelta (23). Sono proposte anche strategie quali la tecnica "sandwich" che comporta la rimozione del rene policistico più complicato prima del trapianto e, se indicata, la nefrectomia del rene residuo dopo il trapianto (24); si affacciano ipotesi di nefrectomia per via laparoscopica, meno invasiva per il paziente (25). Alcuni recenti studi propongono l'embolizzazione arteriosa transcatetere (TAE) quale valida alternativa alla chirurgia tradizionale per ridurre il volume renale prima del trapianto renale (26). Dal punto di vista dei tempi dell'intervento secondo diversi autori la nefrectomia può essere eseguita con buoni risultati contemporaneamente al trapianto (27). Ciascuna strategia trova le sue giustificazioni. I fautori dellintervento preventivo enfatizzano il ruolo negativo dell'ingombro addominale, delle complicanze settiche legate alle cisti renali o degli episodi di macroematuria; per contro l'approccio conservativo permette un mantenimento della diuresi residua e della produzione endogena di eritropoietina, non espone ai rischi correlati allintervento chirurgico di nefrectomia ed a problemi di ipotensione cronica tipica dei pazienti nefrectomizzati. Lintervento di nefrectomia al trapianto riduce il numero degli interventi chirurgici, ma espone a una più prolungata ischemia fredda e al rischio di complicanze emorragiche con possibili ripercussioni sulla ripresa funzionale dell'organo trapiantato. Dai dati raccolti dal nostro questionario emerge un orientamento prevalentemente conservativo nel 92\% dei Centri italiani (nefrectomia solo in presenza di problemi clinici); l'esperienza italiana che pone l'ingombro addominale quale principale indicazione alla nefrectomia (a seguire episodi di rottura di cisti e infezioni urinarie) risulta in linea con i dati riportati dalla letteratura internazionale (23). La percentuale di nefrectomie pre-trapianto varia peraltro da Centro a Centro (stimabile fra il 5 e il 25\%) a conferma di una non totale uniformità di comportamento. Nell'analisi condotta mancano dati standardizzabili per l'indicazione alla nefrectomia, quali le dimensioni dei reni in rapporto al soma del paziente; analogamente non viene riportata l'incidenza di nefrectomie post-trapianto. Nonostante tali limiti lo studio permette un'accettabile visione d'insieme del panorama trapiantologico italiano relativamente alla gestione dei pazienti policistici; emerge come la personalizzazione del trattamento, legata alle caratteristiche dei pazienti e all'esperienza del singolo Centro, può costituire un accettabile criterio nella guida delle scelte operative come confermato dai buoni risultati complessivi (sopravvivenza organo e paziente).

In conclusione $\mathrm{i}$ pazienti con patologia policistica si confermano dei buoni candidati a programmi di trapianto renale con un significativo impatto sulle liste di attesa di trapianto renale; le caratteristiche cliniche individuali condizionano le scelte operative. Pur nell'ambito di una patologia sistemica (alterazioni a carico di altri organi) i risultati post-trapianto sono mediamente superiori a quelli della restante popolazione nefropatica; una corretta gestione pre-trapianto (indagini strumentali pre-trapianto, eventuali approcci di bonifica) possono ridurre il rischio di eventi sfavorevoli nel post-trapianto. Restano auspicabili criteri meglio definiti circa le indicazioni all'intervento di nefrectomia nel periodo di permanenza in lista di attesa.

\section{Riassunto}

Lo studio ha analizzato i criteri di gestione e i risultati dei programmi di trapianto nei pazienti con patologia policistica. Sono stati valutati 11.412 trapianti effettuati in Italia nel periodo gennaio 2002-dicembre 2008 (9995 primi trapianti, 114 trapianti combinati fegato/ rene, 1303 trapianti da donatore vivente). I pazienti policistici costituiscono il $12 \%$ della casistica di primo trapianto da cadavere, il $7 \%$ da vivente, il 25\% di trapianto combinato fegato/rene. Relativamente alla nefrectomia pre-trapianto lo studio ha evidenziato in $32 / 35$ Centri Trapianto un atteggiamento conservativo (intervento solo in presenza di problemi clinici); la principale indicazione è costituita dall'ingombro addominale. L'86\% dei Centri richiede indagini di secondo livello (TC) per lo studio delle dimensioni renali ( $46 \%$ senza, $40 \%$ con mezzo di contrasto). Indagini radiologiche (RM o TC) per la ricerca di malformazioni vascolari cerebrali sono richieste nel $97 \%$ dei Centri ( $74 \%$ sempre, $23 \%$ in presenza di anamnesi familiare per emorragia cerebrale). Nell'ambito dei 9995 primi trapianti (follow-up 16112 mesi) i pazienti policistici presentano una miglior sopravvivenza del graft rispetto ad altre nefropatie 
(a 5 anni 86 rispetto $82 \%, \mathrm{p}<0,01$ ). La mortalità risulta sovrapponibile (a 5 anni 94 rispetto a 92,5\%). Anche nei programmi di trapianto combinato fegato/rene si registra un miglior andamento del rene trapiantato nei pazienti con ADPKD (a 1 anno 92 rispetto a 79\%).

Parole chiave: Malattia policistica autosomica dominante (ADPKD), Trapianto renale, Lista d'attesa, Nefrectomia.

Ringraziamenti: il lavoro è stato possibile grazie alla collaborazione dei 35 Centri Italiani di Trapianto che hanno risposto al questionario proposto.

\section{Indirizzo degli Autori: \\ Dr. Giovanni Mosconi \\ U.O. Nefrologia Dialisi e Trapianto \\ Policlinico S. Orsola-Malpighi \\ Via G. Massarenti 9 \\ 40138 Bologna \\ giovanni.mosconi@libero.it}

\section{Bibliografia}

1. Torres VE, Harris PC, Pirson Y. Autosomal dominant polycystic kidney disease. Lancet 2007; 369(9569): 1287-301.

2. Igarashi P and Somlo S. Genetics and Pathogenesis of Polycystic Kidney Disease. J Am Soc Nephrol 2002; 13: 2384-98.

3. Wilson P. Polycystic Kidney Disease. N Engl J Med 2004; 350: 151-64.

4. Hildebrandt F, Benzing T and Katsanis N. Ciliopathies. N Engl J Med 2011; 364: 1533-43.

5. Hateboer N, v Dijk MA, Bogdanova N, et al. Comparison of phenotypes of polycystic kidney disease types 1 and 2. European PKD1-PKD2 Study Group. Lancet 1999; 353(9147): 103-7.

6. Abbott KC, Agodoa LY. Polycystic kidney disease at endstage renal disease in the United States: patient characteristics and survival. Clin Nephrol 2002; 57 (3): 208-14.

7. Perrone RD, Ruthazer R, Terrin NC. Survival after endstage renal disease in autosomal dominant polycystic kid- ney disease: contribution of extrarenal complications to mortality. Am J Kidney Disease 2001; 38(4): 777-84.

8. Niaudet P. Living donor kidney transplantation in patients with hereditary nephropathies. Nat Rev Nephrol 2010; 6(12): 736-43.

9. Pei Y, Obaji J, Dupuis A et al. Unified criteria for ultrasonographic diagnosis of ADPKD. J Am Soc Nephrol 2009; 20(1): 205-12.

10. Huang E, Samaniego-Picota M, McCune T, Melancon JK, Ugarte R, Kraus E, Womer K, Rabb H, Watnick T. DNA testing for live kidney donors at risk for autosomal dominant polycystic kidney disease. Transplantation 2009; 87(1): 133-7.

11. Jacquet A, Pallet N, Kessler M, et al. Outcomes of renal transplantation in patients with autosomal dominant polycystic kidney disease: a nationwide longitudinal study. Transpl Int 2011; 24(6): 582-87.

12. Andreoni KA, Pelletier RP, Elkhammas EA, et al. Increased incidence of gastrointestinal surgical complications in renal transplant recipients with polycystic kidney disease. Transplantation 1999; 67: 262.

13. Ducloux D, Motte G, Vautrin P, et al. Polycystic kidney disease as a risk factor for post-transplant diabetes mellitus. Nephrol Dial Transplant 1999; 14: 1244.

14. Hamer RA, Chow CL, Ong AC, et al. Polycystic kidney disease is a risk factor for new-onset diabetes after transplantation. Transplantation 2007; 57: 73.

15. Bretagnol A, Halimi JM, Roland M, et al. Autosomal dominant polycystic kidney disease: risk factor for non melanoma skin cancer following kidney transplantation. Transpl Int 2010; 23: 878.

16. Mosconi G, Scolari MP, Feliciangeli G, et al. Nephrological indications in combined liver-kidney transplantation. Transplant Proc 2006; 38(4): 1086-8.

17. Xu HW, Yu SQ, Mei CL, Li MH. Screening for intracranial aneurysm in 355 patients with autosomal-dominant polycystic kidney disease. Stroke 2011; 42(1): 204-6.

18. Gieteling EW, Rinkel GJ. Characteristics of intracranial aneurysms and subarachnoid haemorrhage in patients with polycystic kidney disease. J Neurol 2003; 250(4): 418-23.

19. Brisman JL, Song JK, Newell DW. Cerebral aneurysms. N Engl J Med 2006; 355(9): 928-39.

20. Cohen D, Timsit MO, Chrétien Y, Thiounn N, et al. Place of nephrectomy in patients with autosomal dominant polycystic kidney disease waiting for renal transplantation. Prog Urol 2008; 18(10): 642-9.

21. Bradza E, Ofner D, Riedmann B, et al. The effect of nephrectomy on the outcome of renal transplantation in patients with polycystic kidney disease. Ann Transplant 1996; 1(2): 15-8.

22. Rayner BL, Cassidy MJ, Jacobsen JE, et al. Is preliminary 
binephrectomy necessary in patients with autosomal dominant polycystic kidney disease undergoing renal transplantation? Clin Nephrol 1990; 34(3): 122-4.

23. Kirkman MA, van Dellen D, Mehra S, et al. Native nephrectomy for autosomal dominant polycystic kidney disease: before or after transplantation? BJU International 2010; Dec 16.

24. Cassuto-Viguier E, Quintens H, Chevallier D, et al. Transplantation and nephrectomy in autosomal dominant polycystic disease. Clin Nephrol 1991; 36(2): 105-6.

25. Ismail HR, Flechner SM, Kaouk JH, et al. Simultaneous vs. sequential laparoscopic bilateral native nephrectomy and renal transplantation. Transplatation 2005; 80(8): 1124-7.

26. Cornelis F, Couzi L, Le Bras Y, et al. Embolization of polycystic kidneys a san alternative to nephrectomy before renal transplantation: a pilot study. Am J Transplant 2010; 10(10): 2363-9.

27. Drognitz O, Kirste G, Schramm I, et al. Kidney transplantation with concomitant unilateral nephrectomy: a matched-pair analysis on complications and outcome. Transplantation 2006; 81(6): 874-80. 